\title{
Ticks and tick-borne pathogens on the rise
}

José de la Fuente and Agustín Estrada-Peña

Vector-borne diseases accounted for over 20\% of all emerging infectious diseases recorded from 1940 to 2004 (Jones et al., 2008). The increased risks associated with ticks and tick-borne pathogens (TBPs) are supported by expansion of tick populations into areas where they were previously absent and by some changes in social habits, increasing the contact between humans and ticks (Gray et al., 2009 and Sumilo et al., 2006). Recent upsurges of tick-borne diseases include tick-borne encephalitis (TBE) in Central and Eastern Europe, Kyasanur forest disease (KFD) in Karnataka state of India, Crimean-Congo hemorrhagic fever (CCHF) in northern Turkey and the southwestern regions of the Russian Federation, and Rocky Mountain spotted fever (RMSF) in Arizona and Baja California in the United States (Randolph, 2008, Pattnaik, 2006, Maltezou et al., 2010 and McQuiston et al., 2010). New molecular technologies greatly advanced the study of ticks and TBPs. Newly developed or refined diagnostic tools have increased the efficacy and accuracy for identifying TBPs. New genetic tools and sequencing platforms have improved our possibilities for characterizing ticks and TBPs at the genomic and population levels. Systems biology approaches to the study of ticks and TBPs have resulted in growing databases of transcripts and proteins expressed under different physiological conditions and at the tick-host-pathogen interface. These technologies increase our understanding of ticks and TBPs systematics, genetics, ecology, epidemiology, evolution, and molecular biology, which in turn impact on the development of risk awareness models and control measures. A new approach to characterize the changing landscape of ticks and TBPs is emerging: an integrative framework aimed to join efforts ranging from macro-ecology to molecular biology, from 'omics' to risk management. Recent reports clearly reflect that ticks and TBPs are on the rise, as do their risks for human and animal health worldwide. But fortunately, research and development in this area is also on the rise. Knowledge about ticks and TBPs continues to accumulate in papers published in peer-reviewed journals (Fig. 1), and the perspectives in this field are attracting groups traditionally working with other arthropods. These trends were reflected at the Seventh International Ticks and Tick-borne Pathogens (TTP7) Conference, held in Zaragoza, Spain, August 28thSeptember 2nd, 2011, and in the papers selected for inclusion into this special issue of Ticks and Tick-borne Diseases. The meeting intended to integrate systematics, epidemiology, genetics, and molecular biology research on ticks and TBPs. This objective was fulfilled with 501 accepted papers presented in 8 sessions at the meeting, with the attendance of 320 delegates from 38 countries.

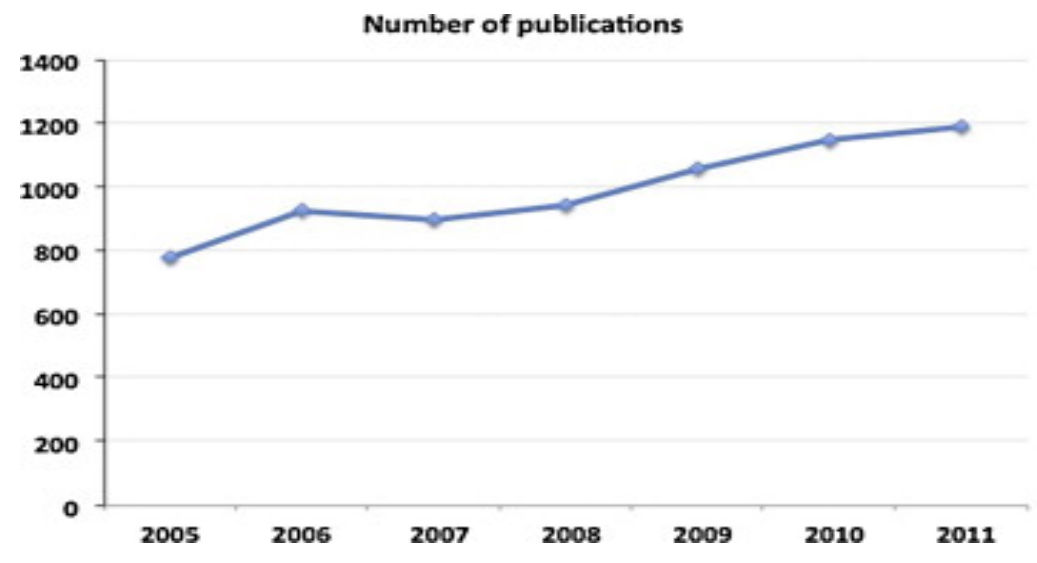


Several conclusions could be drawn from discussions at the meeting that resume the state of the art and future directions in this area:

1. Reports on TBPs continue to increase, but lacking the necessary relationship between reservoir hosts, ticks, and pathogens, which need to be linked together to describe their true eco-epidemiological patterns.

2. It is necessary to switch from local, 'closed-box' epidemiological studies of ticks and TBPs to developing a framework that integrates the information of the different layers of complexity of the system. This approach would allow development of better risk assessment models. Knowledge is accumulating in this direction and several paradigms such as Lyme borreliosis are currently evolving.

3. The development of new in vitro and in vivo animal model systems for ticks and TBPs is growing and will likely continue to grow in the future with an impact on research in this area.

4. While acaricides are still the most widely used control strategy for ticks and TBPs, new control strategies based on the molecular biology and the biological and ecological patterns of both vectors and pathogens are urgently needed.

5. Systems biology approaches to research on ticks and TBPs are generating a huge amount of information, but algorithms need to be developed to use these data for advancing knowledge on basic biological questions and the development of improved vaccines for the control of ticks and TBPs.

6. Although holistic approaches to the study of the interactions between the components of biological systems, and how these interactions give rise to the function and behavior of that system using 'omics' technology are necessary to advance knowledge in these areas, reductionist approaches focusing on specific components of the system are also valuable to address ticks and TBPs genetics, physiology and other biological questions. 